\title{
LINEAR FUNCTIONALS ON THE SMIRNOV CLASS OF THE UNIT BALL IN $C^{n}$
}

\author{
M. Nawrocki
}

\section{Introduction}

Functional analytic properties of many classical spaces of analytic functions have received much attention in recent years. In particular, topological duals of the Hardy spaces of many typical domains have been identified (see $[1,2,5,6,7$, 13]). The Smirnov class $N_{*}$ of the unit disc in the complex plane was extensively studied by $\mathrm{N}$. Yanagihara [16, 17], who described all continuous linear functionals on $N_{*}$ and found multipliers of $N_{*}$ into Hardy spaces. However, up to now, no satisfactory characterization of linear functionals on the Smirnov class $N_{*}(D)$ of any multidimensional domain $D$ has been obtained. The present paper is a study of the linear space structure of the Smirnov class $N_{*}\left(\mathbf{B}_{n}\right)$ of the unit ball $\mathbf{B}_{n}$ in the space of $n$-complex variables $\mathbf{C}^{n}$.

We recall that a function $f$, analytic in $\mathbf{B}_{n}$, is said to be in the Nevanlinna class $N\left(\mathbf{B}_{n}\right)$ if

$$
\sup _{0<r<1} \int_{\partial \mathbf{B}_{n}} \log ^{+}|f(r \zeta)| d \sigma(\zeta)<\infty
$$

where $\sigma$ is the rotation invariant probability measure on $\mathbf{S}=\partial \mathbf{B}_{n}$. The Smirnov class or the Hardy algebra $N_{*}\left(\mathbf{B}_{n}\right)$ is the subspace of $N\left(\mathbf{B}_{n}\right)$ consisting of those functions $f$ for which the family $\left\{\log ^{+}|f(r \cdot)|: 0<r<1\right\}$ is uniformly integrable on $\mathbf{S}$. It is well known that $N_{*}\left(\mathbf{B}_{n}\right)$ equipped with the topology induced by the metric

$$
d(f, g)=\|f-g\|=\lim _{r \rightarrow 1-} \int_{\mathbf{S}} \log (1+|f(r \zeta)-g(r \zeta)|) d \sigma(\zeta)
$$

is an $F$-space (i.e., complete metrizable t.v.s.). For each $f \in N_{*}\left(\mathbf{B}_{n}\right)$, the radial limit $f^{*}(\zeta)=\lim _{r \rightarrow 1-} f(r \zeta)$ exists for almost all $\zeta \in \mathbf{S}$ and

$$
\|f\|=\int_{\mathbf{S}} \log \left(1+\left|f^{*}\right|\right) d \sigma
$$

The reader is referred to [11] for information on $N_{*}\left(\mathbf{B}_{n}\right)$.

In 1976 M. Stoll proved 
Theorem S ([14] Theorem 10). For each continuous linear functional $\gamma$ on $N_{*}\left(\mathbf{B}_{n}\right)$ there exists a unique analytic function $g$ on $\mathbf{B}_{n}$ such that

$$
\gamma(f)=\lim _{\substack{r, \varrho \rightarrow 1-\\ r<e}} \int_{\mathbf{S}} f\left(r \varrho^{-1} \zeta\right) \overline{g(\varrho \zeta)} d \sigma(\zeta)
$$

for all $f \in N_{*}\left(\mathbf{B}_{n}\right)$. Conversely, given any $g$ analytic on $\mathbf{B}_{n}$ for which the limit in (S) exists for all $f \in N_{*}\left(\mathbf{B}_{n}\right)$ (S) defines a continuous linear functional on $N_{*}\left(\mathbf{B}_{n}\right)$.

The main result of the present paper is Theorem 3.1, which together with Corollary 3.5 gives, in terms of the growth restriction of the Fourier coefficients of $g$, a necessary and sufficient condition for an analytic function $g$ to define by (S) a continuous linear functional on $N_{*}\left(\mathbf{B}_{n}\right)$. It turns out that the dual space of $N_{*}\left(\mathbf{B}_{n}\right)$ can be identified with the dual of the Fréchet space (locally convex $F$-space) $F_{*}\left(\mathbf{B}_{n}\right)$ invented by M. Stoll [15] (see Section 2). This will imply that $F_{*}\left(\mathbf{B}_{n}\right)$ is the so called Fréchet envelope of $N_{*}\left(\mathbf{B}_{n}\right)$, i.e., the completion of the space $\left(N_{*}\left(\mathbf{B}_{n}\right), \tau^{c}\right)$, where $\tau^{c}$ is the strongest locally convex topology on $N_{*}\left(\mathbf{B}_{n}\right)$ which is weaker than the original topology $\tau$ of $N_{*}\left(\mathbf{B}_{n}\right)$.

In Section 4 we show that if $n \neq m$, then $F_{*}\left(\mathbf{B}_{n}\right)$ is not isomorphic to $F_{*}\left(\mathbf{B}_{m}\right)$. This implies that the Smirnov classes $N_{*}\left(\mathbf{B}_{n}\right), N_{*}\left(\mathbf{B}_{m}\right)$ are not isomorphic for different dimensions $n, m$. The results of the Sections 3 and 4 are applied to obtain the best possible estimate of Fourier coefficients of functions in $N_{*}\left(\mathbf{B}_{n}\right)$.

\section{Preliminaries}

Throughout the paper we use the standard notation of [11]. Let $\langle z, w\rangle=$ $\sum_{j=1}^{n} z_{j} \bar{w}_{j}\left(z, w \in \mathbf{C}^{n}\right)$ denote the standard inner product on $\mathbf{C}^{n}$ and $|z|=$ $\langle z, z\rangle^{1 / 2}\left(z \in \mathbf{C}^{n}\right)$ the corresponding norm on $\mathbf{C}^{n}$. We denote the unit ball and the unit sphere in $\mathbf{C}^{n}$ by $\mathbf{B}=\mathbf{B}_{n}$ and $\mathbf{S}=\mathbf{S}_{n}=\partial \mathbf{B}_{n}$ respectively. Moreover, let $\mathbf{Z}_{+}$denote the set of all nonnegative integers and $\mathbf{Z}_{+}^{n}$ its $n$-fold product. $\mathbf{T}^{n}$ is the $n$-fold product of $\mathbf{T}=\partial \mathbf{B}_{1}$.

For any multi-index $\alpha=\left(\alpha_{1}, \ldots, \alpha_{n}\right) \in \mathbf{Z}_{+}^{n}$ and $z, \zeta \in \mathbf{C}^{n}, r \in \mathbf{C}$

$$
\begin{aligned}
|a| & :=\alpha_{1}+\cdots+\alpha_{n}, \quad z \zeta:=\left(z_{1} \zeta_{1}, \ldots, z_{n} \zeta_{n}\right), \\
\alpha ! & :=\alpha_{1} ! \cdots \alpha_{n} !, \quad r z:=\left(r z_{1}, \ldots, r z_{n}\right), \\
z^{\alpha} & :=z_{1}^{\alpha_{1}} \cdots z_{n}^{\alpha_{n}} .
\end{aligned}
$$

It is well known that the analytic monomials $z^{\alpha}, \alpha \in \mathbf{Z}_{+}^{n}$, are orthogonal on the sphere, i.e.,

$$
\int_{\mathbf{S}} \zeta^{\alpha} \bar{\zeta}^{\beta} d \sigma(\zeta)=0 \quad \text { if } \alpha \neq \beta
$$


Moreover,

$$
\int_{\mathbf{S}}\left|\zeta^{\alpha}\right|^{2} d \sigma(\zeta)=\frac{(n-1) ! \alpha !}{(n-1+|\alpha|) !}
$$

(see [11] 1.4.8, 1.4.9). Therefore,

$$
\varphi_{\alpha}(z)=\left(\frac{(n-1+|\alpha|) !}{(n-1) ! \alpha !}\right)^{1 / 2} z^{\alpha}, \quad \alpha \in \mathbf{Z}_{+}^{n},
$$

is such an orthogonal system of monomials on $\mathbf{B}_{n}$ which is normalized in $L_{2}(\mathbf{S}, \sigma)$.

Each analytic function $f$ on $\mathbf{B}_{n}$ has the Fourier expression

$$
f(z)=\sum_{\alpha \in \mathbf{Z}_{+}^{n}} a_{\alpha}(f) \varphi_{\alpha}(z)
$$

where the series is convergent uniformly and absolutely on each compact subset of $\mathbf{B}_{n}$ and

$$
a_{\alpha}(f)=\lim _{r \rightarrow 1-} \int_{\mathbf{S}} f(r \zeta) \overline{\varphi_{\alpha}(\zeta)} d \sigma(\zeta) .
$$
which

M. Stoll [15] defined the space $F_{*}\left(\mathbf{B}_{n}\right)$ of all analytic functions $f$ on $\mathbf{B}_{n}$ for

$$
\|f\|_{k}=\sup _{\alpha \in \mathbf{Z}_{+}^{n}}\left|a_{\alpha}(f)\right| \exp \left(-|\alpha|^{n /(n+1)} / k\right)<\infty \quad \text { for all } k \in \mathbf{N} .
$$

$F_{*}\left(\mathbf{B}_{n}\right)$ equipped with the topology determined by the sequence of norms $\left\{\|\cdot\|_{k}\right.$ : $k \in \mathbf{N}\}$ is a Fréchet space. Moreover,

Theorem 2.1 ([15] Theorem 5.2).

(a) $N_{*}\left(\mathbf{B}_{n}\right)$ is a dense subspace of $F_{*}\left(\mathbf{B}_{n}\right)$,

(b) the inclusion mapping $N_{*}\left(\mathbf{B}_{n}\right) \rightarrow F_{*}\left(\mathbf{B}_{n}\right)$ is continuous.

\section{Representations of linear functionals}

In this section we prove the following main result of the paper.

Theorem 3.1. If $\left\{b_{\alpha}\right\}_{\alpha \in \mathbf{Z}_{+}^{n}}$ is such a sequence of complex numbers that

$$
b_{\alpha}=O\left(\exp \left(-c|\alpha|^{n /(n+1)}\right)\right) \quad \text { for some } c>0 \text {, }
$$

then

$$
\gamma(f)=\sum a_{\alpha}(f) b_{\alpha}, \quad f \in N_{*}\left(\mathbf{B}_{n}\right),
$$

defines a continuous linear functional $\gamma$ on $N_{*}\left(\mathbf{B}_{n}\right)$ with the convergence in (3.2) being absolute.

Conversely, for each continuous linear functional $\gamma$ on $N_{*}\left(\mathbf{B}_{n}\right)$ there exists a unique sequence $\left\{b_{\alpha}\right\}$ such that (3.1) and (3.2) hold. 
In the proof of this theorem we will use properties of the functions

$$
f_{c, w}(z)=\exp \left(c \frac{1-|w|^{2}}{(1-\langle z, w\rangle)^{n+1}}\right)
$$

where $w, z \in \mathbf{B}_{n}$ and $c>0$.

Lemma 3.2. We have

$$
\lim _{c \rightarrow 0} \sup _{w \in \mathbf{B}}\left\|c f_{c, w}\right\|=0
$$

Proof. In the proof we follow [3]. The measure $\sigma$ on $\mathbf{S}$ is invariant with respect to the group $U(n)$ of all unitary operators of the Hilbert space $\mathbf{C}^{n}$, so $\left\|f_{c, w}\right\|=\left\|f_{c,|w| e_{1}}\right\|$, where $e_{1}$ is the first unit vector in $\mathbf{C}^{n}$. Therefore, it is enough to show that

$$
\lim _{c \rightarrow 0} \sup _{r \in(0,1)}\left\|c f_{c, r e_{1}}\right\|=0 .
$$

Let $U$ be a neighbourhood of $e_{1}$ on $\mathbf{S}$. It is easy to see that

$$
\lim _{c \rightarrow 0} \sup _{\zeta \in \mathbf{S} \backslash U} \sup _{r \in(0,1)}\left|c f_{c, r e(\zeta)}^{*}(\zeta)\right|=0
$$

Consequently,

$$
\lim _{c \rightarrow 0} \sup _{r \in(0,1)} \int_{\mathbf{S} \backslash U} \sup \left(1+\left|c f_{c, r e_{1}}^{*}\right|\right) d \sigma=0 .
$$

Using the inequality $\log (1+c x) \leq \log (1+c)+\log 2+\log x, x \geq 1, c \geq 0$, with $x=\exp \left(c\left(1-r^{2}\right)\left|1-r z_{1}\right|^{-(n+1)}\right)$, we have

$$
\begin{aligned}
\int_{U} \log \left(1+\left|c f_{c, r e_{1}}^{*}\right|\right) d \sigma \leq & \sigma(U) \log (1+c)+\sigma(U) \log 2 \\
& +c \int_{U}\left(1-r^{2}\right)\left|1-r \zeta_{1}\right|^{-(n+1)} d \sigma(\zeta) .
\end{aligned}
$$

However, $\sup _{w \in \mathbf{B}} \int_{\mathbf{S}}\left(1-|w|^{2}\right)|1-\langle w, \zeta\rangle|^{-(n+1)} d \sigma(\zeta)=: C<\infty$ (see [11], 1.4.10), so

$$
\int_{U} \log \left(1+\left|c f_{c, r e_{1}}^{*}\right|\right) d \sigma \leq 2 \sigma(U) \log 2+c C
$$

for any $U$ and $0<c \leq 1$. This completes the proof, because we could choose $U$ at the very beginning as small as we want. 
Lemma 3.3. For each $c>0$ there exists a $k \in \mathbf{N}$ such that

$$
\inf _{\alpha \in \mathbf{Z}_{+}^{n}} \sup _{w \in \mathbf{B}}\left|a_{\alpha}\left(f_{c, w}\right)\right| \exp \left(-|\alpha|^{n /(n+1) j} / k\right)>0 .
$$

Proof. For each $c>0, w, z \in \mathbf{B}$ we have

$$
\begin{aligned}
f_{c, w}(z) & =\sum_{j=0}^{\infty} \frac{c^{j}}{j !}\left(1-|w|^{2}\right)^{j}(1-\langle z, w\rangle)^{-(n+1) j} \\
& =1+\sum_{j=1}^{\infty} \frac{c^{j}}{j !}\left(1-|w|^{2}\right)^{j} \sum_{k=0}^{\infty}\left(\begin{array}{c}
(n+1) j+k-1 \\
k
\end{array}\right)\langle z, w\rangle^{k} \\
& =1+\sum_{j=1}^{\infty} \frac{c^{j}}{j !}\left(1-|w|^{2}\right)^{j} \sum_{k=0}^{\infty}\left(\begin{array}{c}
(n+1) j+k-1 \\
k
\end{array}\right) \sum_{|\alpha|=k} \frac{k !}{\alpha !} z^{\alpha} \bar{w}^{\alpha} \\
& =1+\sum_{k=0}^{\infty} \sum_{|\alpha|=k}\left(\sum_{j=1}^{\infty} \frac{c^{j}}{j !}\left(1-|w|^{2}\right)^{j}\left(\begin{array}{c}
(n+1) j+k-1 \\
k
\end{array}\right) \frac{k !}{\alpha !}\right) \bar{w}^{\alpha} z^{\alpha} \\
& =1+\sum_{k=0}^{\infty} \sum_{|\alpha|=k}\left(\sum_{j=1}^{\infty} v_{j}(c,|w|, k) \frac{|\alpha| !}{\alpha !}\left(\frac{(n-1) ! \alpha !}{(n-1+|\alpha|) !}\right)^{1 / 2}\right) \bar{w}^{\alpha} \varphi_{\alpha}(z)
\end{aligned}
$$

where

$$
v_{j}(c,|w|, k):=\frac{c^{j}}{j !}\left(1-|w|^{2}\right)^{j}\left(\begin{array}{c}
(n+1) j+k-1 \\
k
\end{array}\right) .
$$

Thus, if $\mu(c, \alpha):=\sup _{w \in \mathbf{B}}\left|a_{\alpha}\left(f_{c, w}\right)\right|$, then

$$
\mu(c, \alpha) \geq v_{j}(c,|w|,|\alpha|) \frac{|\alpha| !}{\alpha !}\left(\frac{(n-1) ! \alpha !}{(n-1+|\alpha|) !}\right)^{1 / 2} \bar{w}^{\alpha}
$$

for all $j \in \mathbf{N}, w=\left(w_{1}, \ldots, w_{n}\right) \in \mathbf{B}$ with $w_{i} \leq 0, i=1,2, \ldots, n$, and all $\alpha \in \mathbf{Z}_{+}^{n} \backslash\{0\}$. However, by (2.1), for every $\alpha \in \mathbf{Z}_{+}^{n}$ there is a $\xi \in \mathbf{S}$ such that $\left|\xi^{\alpha}\right|=((n-1) ! \alpha ! /(n-1+|\alpha|) !)^{1 / 2}$. Of course, we can choose $\xi=\left(\xi_{1}, \ldots, \xi_{n}\right)$ such that $\xi_{i} \geq 0, i=1,2, \ldots, n$. Consequently, taking $w:=r \xi$, where $r \in(0,1)$, we obtain

$$
\mu(c, \alpha) \geq v_{j}(c, r,|\alpha|) \frac{(n-1) !}{(|\alpha|+1) \cdots(|\alpha|+n-1)} r^{|\alpha|}
$$

for all $j \in \mathbf{N}, r \in(0,1), \alpha \in \mathbf{Z}_{+}^{n} \backslash\{0\}$. Using the obvious inequality 


$$
\left(\begin{array}{c}
(n+1) j+k-1 \\
k
\end{array}\right) \geq \frac{k^{(n+1) j-1}}{((n+1) j) !}
$$

we have

$$
\mu(c, \alpha) \geq \frac{c^{j}}{j !}\left(1-r^{2}\right)^{j} \frac{|\alpha|^{(n+1) j}}{((n+1) j !)} \frac{r^{|\alpha|}}{|\alpha|(|\alpha|+1) \cdots(|\alpha|+n-1)}
$$

for all $j \in \mathbf{N}, r \in(0,1), \alpha \in \mathbf{Z}_{+}^{n} \backslash\{0\}$.

Now, let us fix $0<d<\frac{1}{2}$ so small that $d^{n+1}<c(n+1)^{-(n+1)}$. For $\alpha \in$ $\mathbf{Z}_{+}^{n} \backslash\{0\}$ take $j=j_{\alpha}:=$ the integer part of $d|\alpha|^{n /(n+1)}$ and take $r^{2}=r_{\alpha}^{2}:=1-x_{\alpha}$, where $x=x_{\alpha}:=d /|a|^{n /(n+1)}$. Note that $j_{\alpha} \geq 1$ for sufficiently large $|\alpha|$ as well as $r_{\alpha} \in\left(0, \frac{1}{2}\right)$.

Using Stirling's formula we have

$$
\begin{aligned}
\log \mu(c, \alpha) & \geq j \log c+j \log \left(1-r^{2}\right)+j \log |\alpha|^{n+1}+|\alpha| \log r-j \log j+j-O(\log j) \\
& -j \log ((n+1) j)^{n+1}+(n+1) j-O(\log (n+1) j)-O(\log |\alpha|) \\
& =j\left(n+2+\log \frac{c\left(1-r^{2}\right)|\alpha|^{n+1}}{(n+1)^{n+1} j^{n+2}}\right)+|\alpha| \log r-O(\log |\alpha|) .
\end{aligned}
$$

However,

$$
\frac{c\left(1-r^{2}\right)|\alpha|^{n+1}}{(n+1)^{n+1} j^{n+2}} \geq \frac{c d|\alpha|^{n+1-1 /(n+1)}}{(n+1)^{n+1} d^{n+2}|\alpha|^{(n+2) n /(n+1)}} \geq 1
$$

for large $\alpha$ and

$$
|\alpha| \log r=\frac{1}{2}|\alpha| \log (1-x) \geq-|\alpha| x=-d|\alpha|^{n /(n+1)},
$$

since $0<x<\frac{1}{2}$. Finally,

$$
\begin{aligned}
\log \mu(c, \alpha) & \geq(n+2)\left(d|\alpha|^{n /(n+1)}\right)-d|\alpha|^{n /(n+1)}-O(\log |\alpha|) \\
& \geq|\alpha|^{n /(n+1)} / k-O(\log |\alpha|)
\end{aligned}
$$

for some $k \in \mathbf{N}$. The proof is completed.

Lemma 3.4. For each $n, k \in \mathbf{N}$ and each family of complex numbers $\left\{x_{\alpha}\right.$ : $\left.\alpha \in \mathbf{Z}_{+}^{n},|\alpha|=k\right\}$ we have

$$
\max _{\alpha}\left|x_{\alpha}\right| \geq \max _{\xi \in \mathbf{T}^{n}}\left|\sum_{\alpha} x_{\alpha} \xi^{\alpha}\right|
$$


Proof. Let $m_{n}$ be the normalized Lebesgue measure on $\mathbf{T}$. The system of monomials $\xi^{\alpha}, \alpha \in \mathbf{Z}_{+}^{n}$, is ortonormal in $L_{2}\left(\mathbf{T}^{n}, m_{n}\right)$, so

$$
\max _{\xi \in \mathbb{T}^{n}}\left|\sum_{|\alpha|=k} x_{\alpha} \xi^{\alpha}\right|^{2} \geq \int_{\mathbf{T}^{n}}\left|\sum_{\alpha} x_{\alpha} \xi^{\alpha}\right|^{2} d m_{n}(\xi)=\sum_{\alpha}\left|x_{\alpha}\right|^{2} \geq \max _{\alpha}\left|x_{\alpha}\right|^{2} .
$$

Proof of Theorem 3.1. M. Stoll has proved that (3.2) with $\left\{b_{\alpha}\right\}$ satisfying (3.1) is a representation of continuous linear functionals on the space $F_{*}\left(\mathbf{B}_{n}\right)$ (see [15] Theorem 5.3). This and Theorem 2.1 imply that each $\left\{b_{\alpha}\right\}$ satisfying (3.1) defines by (3.2) a continuous linear functional on $N_{*}\left(\mathbf{B}_{n}\right)$.

Suppose now that $\gamma$ is a continuous linear functional on $N_{*}\left(\mathbf{B}_{n}\right)$. Put $b_{\alpha}:=$ $\gamma\left(\varphi_{\alpha}\right)$ for $\alpha \in \mathbf{Z}_{+}^{n}$. Then there is an $\varepsilon>0$ such that

$$
|\gamma(f)| \leq 1 \quad \text { for all } f \in N_{*}\left(\mathbf{B}_{n}\right), \quad\|f\|<\varepsilon .
$$

For each $f \in N_{*}\left(\mathbf{B}_{n}\right)$ and $\zeta \in \mathbf{D}=\mathbf{B}_{1}$ we have $\|f(\zeta \cdot)\| \leq\|f\|$ and

$$
\gamma_{f}(\zeta):=\gamma(f(\zeta \cdot))=\sum_{k=0}^{\infty}\left(\sum_{|\alpha|=k} a_{\alpha}(f) b_{\alpha}\right) \zeta^{k} .
$$

Therefore, for each $f \in N_{*}\left(\mathbf{B}_{n}\right),\|f\| \leq \varepsilon$, the function $\gamma_{f}$ is analytic on the unit $\operatorname{disc} \mathbf{D}$ in $\mathbf{C}$ and $\sup _{\zeta \in \mathbf{D}}\left|\gamma_{f}(\zeta)\right| \leq 1$. Consequently,

$$
\left|\sum_{|\alpha|=k} a_{\alpha}(f) b_{\alpha}\right| \leq 1 \quad \text { for all } f \in N_{*}(\mathbf{B}),\|f\| \leq \varepsilon, k \in \mathbf{N} .
$$

Lemma 3.2 tells us that there exists $a c>0$ such that

$$
\left\|c f_{c, w}\right\| \leq \varepsilon \quad \text { for all } w \in \mathbf{B} .
$$

Moreover, by Lemma 3.3, there are $k \in \mathbf{N}, \delta>0$ such that

$$
\sup _{w \in \mathbf{B}}\left|a_{\alpha}(f c, w)\right| \geq \delta \exp \left(|\alpha|^{n /(n+1)} / k\right) \quad \text { for all } \alpha \in \mathbf{Z}_{+}^{n} .
$$

Let us fix an arbitrary $\beta \in \mathbf{Z}_{+}^{n}$. By (3.7) we can choose such a $w=w_{\beta} \in \mathbf{B}$ that

$$
\left|a_{\beta}\left(f_{c, w}\right)\right| \geq \delta \exp \left(|b|^{n /(n+1)} / k\right) .
$$

Using Lemma 3.4 we can find $\xi \in \mathbf{T}^{n}$ such that

$$
\left|a_{\beta}\left(f_{c, w}\right) b_{\beta}\right| \leq\left|\sum_{|\alpha|=|\beta|} a_{\beta}\left(f_{c, w}\right) b_{\alpha} \xi^{\alpha}\right| .
$$


Let us observe that $\left\|c f_{c, w}(\xi \cdot)\right\|=\left\|c f_{c, w}\right\| \leq \varepsilon$ and $f_{c, w}(\xi z)=\sum_{\alpha} \alpha\left(f_{c, w}\right) \xi^{\alpha} \varphi_{\alpha}(z)$, $z \in \mathbf{B}$. Therefore, (3.9) and (3.5) imply

$$
\left|c a_{\beta}\left(f_{c, w}\right) b_{\beta}\right| \leq\left|\sum_{|\alpha|=|\beta|} a_{\alpha}\left(c f_{c, w}(\xi \cdot)\right) b_{\alpha}\right| \leq 1 .
$$

Finally, by (3.8),

$$
\left|b_{\beta}\right| \leq\left|c a_{\beta}\left(f_{c, w}\right)\right| \leq(c \delta)^{-1} \exp \left(-|\beta|^{n /(n+1)} / k\right) .
$$

We have proved that $\left\{b_{\alpha}\right\}$ satisfies (3.1). By the first part of the theorem, $\left\{b_{\alpha}\right\}$ defines by $\Gamma(f)=\sum a_{\alpha}(f) b_{\alpha}$ a continuous linear functional on $N_{*}\left(\mathbf{B}_{n}\right)$, which coincides with $\gamma$ on the space of all polynomials. Finally, $\Gamma(f)=\gamma(f)$ for all $f \in N_{*}\left(\mathbf{B}_{n}\right)$. The proof is finished.

Corollary 3.5. An analytic function $g(z)=\sum b_{\alpha} \varphi_{\alpha}(z), z \in \mathbf{B}_{n}$, defines by (S) a continuous linear functional on $N_{*}\left(\mathbf{B}_{n}\right)$ if and only if $\left\{b_{\alpha}\right\}$ satisfies (3.1).

Proof. The absolute convergence of the series $\sum a_{\alpha}(f) \bar{b}_{\alpha}, f \in N_{*}\left(\mathbf{B}_{n}\right)$, is easily seen to imply that the limit in (S) exists and is equal to the sum of this series. Therefore, we see, by Theorem 3.1, that if $\left\{b_{\alpha}\right\}$ satisfies (3.1) then the analytic function $g(z)=\sum b_{\alpha} \varphi_{\alpha}(z)$ defines by (S) a continuous linear functional on $N_{*}\left(\mathbf{B}_{n}\right)$.

Conversely, if $\gamma$ is a continuous linear functional on $N_{*}\left(\mathbf{B}_{n}\right)$ defined by (S) with $g(z)=\sum b_{\alpha} \varphi_{\alpha}(z)$, then

$$
\gamma(f)=\sum a_{\alpha}(f) \bar{b}_{\alpha} \quad \text { for each polynomial } f .
$$

The set of polynomials is dense in $N_{*}\left(\mathbf{B}_{n}\right)$, so the equality in (3.10) holds for all $f \in N_{*}\left(\mathbf{B}_{n}\right)$ and $\left\{b_{\alpha}\right\}$ satisfies (3.1).

Let us recall that if $X=(X, \tau)$ is an $F$-space whose topological dual $X^{\prime}$ separates the points of $X$, its Fréchet envelope $\hat{X}$ is defined to be the completion of the space $\left(X, \tau^{C}\right)$, where $\tau^{C}$ is the strongest locally convex topology on $X$ which is weaker than $\tau$. In fact it is known (see [13]) that $\tau^{C}$ is equal to the Mackey topology of the dual pair $\left(X, X^{\prime}\right)$. Since for each locally convex, metrizable topology $\xi$ on $X,(X, \xi)$ is a Mackey space, i.e., $\xi$ coincides with the Mackey topology of the dual pair $\left(X, X_{\xi}^{\prime}\right)$ (cf. [12] Chapter IV. 3.4), so the Fréchet envelope $\hat{X}$ of $X$ is up to an isomorphism uniquely defined by

(FE1) $\hat{X}$ is a Fréchet space,

(FE2) there exists a continuous embedding $j$ of $X$ onto a dense subspace of $\hat{X}$, (FE3) the mapping $\gamma \mapsto \gamma \circ j$ is a linear isomorphism of $\hat{X}^{\prime}$ onto $X^{\prime}$.

Theorem 3.7. The space $F_{*}\left(\mathbf{B}_{n}\right)$ is the Frèchet envelope of $N_{*}\left(\mathbf{B}_{n}\right)$.

Proof. Let $j$ be the inclusion mapping of $N_{*}\left(\mathbf{B}_{n}\right)$ into $F_{*}\left(\mathbf{B}_{n}\right)$. Then (FE2) holds because of Theorem 2.1 while (FE3) is a consequence of Theorem 3.1 and [15] Theorem 5.3. 


\section{The nuclearity of $F_{*}\left(\mathbf{B}_{n}\right)$ and applications}

We recall that a nondecreasing sequence $\gamma=\left\{\gamma_{j}\right\}$ is said to be a stable nuclear exponential sequence of finite type if $\sup \gamma_{2 j} / \gamma_{j}<\infty$ and $\lim (\log j) / \gamma_{j}=0$. For each such sequence $\gamma$ the finite type power series space $\Lambda_{1}(\gamma)$ is defined to be the space of all complex sequences $x=\left\{x_{j}\right\}$ such that

$$
q_{k}(x)=\sup \left|x_{j}\right| \exp \left(-\gamma_{j} / k\right)<\infty \quad \text { for each } k \in \mathbf{N}
$$

(see [4]). It is well known that $\Lambda_{1}(\gamma)$ is nuclear.

We say that two sequences $\gamma, \gamma^{\prime}$ are equivalent $\left(\gamma \sim \gamma^{\prime}\right)$ if

$$
0<\liminf _{j}\left(\gamma_{j} / \gamma_{j}^{\prime}\right) \leq \limsup _{j}\left(\gamma_{j} / \gamma_{j}^{\prime}\right)<\infty .
$$

Proposition 4.1. For every $n \in \mathbf{N}$ the space $F_{*}\left(\mathbf{B}_{n}\right)$ is isomorphic to $\Lambda_{1}\left(j^{1 /(n+1)}\right)$. Consequently, if $m>n$, there does not exist any subspace of $F_{*}\left(\mathbf{B}_{n}\right)$ isomorphic to $F_{*}\left(\mathbf{B}_{m}\right)$.

Proof. Let $j$ be any bijection of $\mathbf{Z}_{+}^{n}$ onto $\mathbf{Z}_{+}$such that $j(\alpha)<j\left(\alpha^{\prime}\right)$ whenever $|\alpha|<\left|\alpha^{\prime}\right|$. It is easily seen that $j(\alpha) \sim|\alpha|^{n}$. Consequently, the operator $T$ : $F_{*}\left(\mathbf{B}_{n}\right) \rightarrow \Lambda_{1}\left(j^{1 /(n+1)}\right)$ defined by $T(f):=\left\{x_{j}(f)\right\}$, where $x_{j}(f):=a_{\alpha}(f)$ if $j=j(\alpha)$, is a linear and topological isomorphism.

The second assertion of the proposition immediately follows from the first one and Proposition 3 in [4].

Theorem 4.2. If $n<m$, there is no complemented subspace of $N_{*}\left(\mathbf{B}_{n}\right)$ isomorphic to $N_{*}\left(\mathbf{B}_{m}\right)$. In particular, the spaces $N_{*}\left(\mathbf{B}_{n}\right)$ and $N_{*}\left(\mathbf{B}_{m}\right)$ are not isomorphic.

Proof. Suppose that $P$ is a continuous projection of $N_{*}\left(\mathbf{B}_{n}\right)$ onto its subspace $X$ isomorphic to $N_{*}\left(\mathbf{B}_{m}\right) . \quad P$ remains continuous if we equip $N_{*}\left(\mathbf{B}_{n}\right)$ and $X$ with their own Mackey topologies. $X$ is complemented in $N_{*}\left(\mathbf{B}_{n}\right)$, so the Mackey topology of $X$ coincides with the topology induced on $X$ by the Mackey topology of the whole space $N_{*}\left(\mathbf{B}_{n}\right)$. Consequently, the extension of $P$ to the Fréchet envelope $F_{*}\left(\mathbf{B}_{n}\right)$ of $N_{*}\left(\mathbf{B}_{n}\right)$ is a continuous projection of $F_{*}\left(\mathbf{B}_{n}\right)$ onto the closure $\bar{X}$ of $X$ in $F_{*}\left(\mathbf{B}_{n}\right)$. Finally, $\bar{X}$ is a subspace of $F_{*}\left(\mathbf{B}_{n}\right)$ which is isomorphic to $\hat{X} \simeq F_{*}\left(\mathbf{B}_{m}\right)$. This contradicts Proposition 4.1.

M. Stoll proved that $a_{\alpha}(f)=O\left(\exp \left(o(\alpha)|\alpha|^{n /(n+1)}\right)\right)$ is the best possible estimate of Fourier coefficients of functions $f$ in $F_{*}\left(\mathbf{B}_{n}\right)$ (see [15] Theorem 5.1). We apply the nuclearity of $F_{*}\left(\mathbf{B}_{n}\right)$ to show that this estimate is the best possible one for $N_{*}\left(\mathbf{B}_{n}\right)$.

Proposition 4.4. For each sequence of positive numbers $\left\{\lambda_{\alpha}\right\}$ decreasing to zero there is an $f \in N_{*}\left(\mathbf{B}_{n}\right)$ such that

$$
\sup \left\{\left|a_{\alpha}(f)\right| \exp \left(-\lambda_{\alpha}|\alpha|^{n /(n+1)}\right): \alpha \in \mathbf{Z}_{+}^{n}\right\}=\infty \text {. }
$$


Proof. Our proof follows [8] Section 4. Suppose that

$$
a_{\alpha}(f)=O\left(\exp \left(\lambda_{\alpha}|\alpha|^{n /(n+1)}\right)\right.
$$

for all $f \in N_{*}\left(\mathbf{B}_{n}\right)$. Let $X$ be the Banach space of all analytic functions on $\mathbf{B}_{n}$ for which

$$
\|f\|=\sup _{\alpha}\left|a_{\alpha}(f)\right| \exp \left(-\lambda_{\alpha}|\alpha|^{n /(n+1)}\right)<\infty .
$$

It is easily seen that the topologies of $X, F_{*}\left(\mathbf{B}_{n}\right)$, and $N_{*}\left(\mathbf{B}_{n}\right)$ are stronger than the weak topologies defined on these spaces by the continuous linear functionals $f \rightarrow a_{\alpha}(f), \alpha \in \mathbf{Z}_{+}^{n}$. Moreover, $X \subseteq F_{*}\left(\mathbf{B}_{n}\right)$ and, of course, $N_{*}\left(\mathbf{B}_{n}\right) \subseteq X$. Consequently, both inclusion above mappings have closed graphs, and so, by the closed graph theorem, they are continuous. Therefore, the topology of $X$ restricted to $N_{*}\left(\mathbf{B}_{n}\right)$ coincides with the topology induced on $N_{*}\left(\mathbf{B}_{n}\right)$ by $F_{*}\left(\mathbf{B}_{n}\right)$ (the strongest locally convex topology on $N_{*}\left(\mathbf{B}_{n}\right)$ which is weaker than the original topology of $\left.N_{*}\left(\mathbf{B}_{n}\right)\right)$. Consequently, the closure of $N_{*}\left(\mathbf{B}_{n}\right)$ in $X$ is the Fréchet envelope of $N_{*}\left(\mathbf{B}_{n}\right)$. However, this is impossible, because each nuclear Banach space is finite dimensional.

\section{References}

[1] Aleksandrov, A.B.: Essay on non locally convex Hardy classes. - Lecture Notes in Mathematics 864. Springer-Verlag, Berlin-Heidelberg-New York, 1981, 1-89.

[2] Colfman, R.P., and R. Rochberg: Representation theorems for holomorphic and harmonic functions in $L_{p}$. - Astérisque, 77, 1980, 11-66.

[3] Drewnowski, L.: Topological vector groups and the Nevanlinna class. - Preprint.

[4] Dubinsky, E.: Basic sequences in stable finite type power series spaces. - Studia Math. $68,1980,117-130$.

[5] Duren, P.L., B.W. Romberg, and A.L. Shields: Linear functionals of $H^{p}$ with $0<$ $p<1$. - J. Reine Angew. Math. 238, 1969, 32-60.

[6] Frazier, A.P.: The dual space of $H^{p}$ of the polydisc for $0<p<1$. - Duke Math. J. 39 $1972,369-373$.

[7] HahN, K.T., and J. Mitchel: Representation of linear functionals in $H^{p}$ spaces over bounded symmetric domains in $\mathbf{C}^{n}$. - J. Math. Anal. Appl. 56, 1976, 379-396.

[8] Nawrocki, M.: The Fréchet envelopes of the vector-valued Smirnov classes. - Studia Math. 94, 1989 (to appear).

[9] PRIVALOV, I.I.: Boundary properties of analytic functions. - GITTL, Moscow, 1950. German translation: Rand-eigenschaften analytischer Funktionen. VEB Deutscher Verlag der Wissenschaften, Berlin, 1956.

[10] Rolewicz, S.: Metric linear spaces. - PWN-Polish Scientific Publisher, Warszawa; D. Reidel Publishing Company, Dordrecht-Boston-Lancaster, 1984.

[11] Rudin, W.: Function theory in the unit ball of $\mathbf{C}^{n}$. - Springer-Verlag, Berlin-HeidelbergNew York, 1980.

[12] Shaeffer, H.H.: Topological vector spaces. - Springer-Verlag, New York-HeidelbergBerlin, 1971. 
[13] ShaPIRo, J.H.: Mackey topologies, reproducing kernels, and diagonal maps on Hardy and Bregman spaces. - Duke Math. J. 43, 1976, 187-202.

[14] Stoll, M.: The space $N_{*}$ of holomorphic functions on bounded symmetric domains. Ann. Polon. Math. 32, 1976, 95-110.

[15] Stoll, M.: Mean growth and Fourier coefficients of some classes of holomorphic functions on bounded symmetric domains. - Ann. Polon. Math. 45, 1985, 161-183.

[16] Yanagihara, N.: The containing Fréchet space for the class $\mathrm{N}^{+}$. - Duke Math. J. 40, 1973, 93-103.

[17] Yanagihara, N.: Multipliers and linear functionals for the class $N^{+}$. - Trans. Amer. Math. Soc. 180, 1973, 449-461.

[18] Yanagihara, N.: Mean growth and Taylor coefficients of some classes of functions. - Ann. Polon. Math. 30, 1974, 37-48.

\author{
A. Mickiewicz University \\ Institute of Mathematics \\ Ul. Matejki $48 / 49$ \\ PL-60-769 Poznan \\ Poland
}

Received 11 November 1988 